\title{
Capture of Perfluorooctanoic Acid using Oil-Filled Graphene Oxide-Silica Hybrid Capsules
}

Muthana Ali, ${ }^{\dagger}$ Shane P. Meaney, ${ }^{\dagger}$ Luke W. Giles, $^{\dagger}$ Phillip Holt, ${ }^{\dagger}$ Mainak Majumder,,

and Rico F. Tabor ${ }^{*}, \dagger$

†School of Chemistry, Monash University, Clayton, VIC 3800, Australia ¥Department of Chemistry, Karbala University, Karbala 56001, Iraq đNanoscale Science and Engineering Laboratory (NSEL), Department of Mechanical and Aerospace Engineering, Monash University Clayton, VIC-3800, Australia

\section{E-mail: rico.tabor@monash.edu}

Phone: +61 39905 4558. Fax: +6139905 4597

This document contains:

11 pages (including this cover page)

8 Figures

7 Tables 



Figure S1. (a) XPS survey spectrum of pure $\mathrm{GO}, \mathrm{SiO}_{2}-\mathrm{GO}$ microcapsules, and $\mathrm{NH}_{2}-$ $\mathrm{SiO}_{2}-\mathrm{GO}$ microcapsules, (b \& c) $\mathrm{C}$ 1s and $\mathrm{Si} 2 \mathrm{p}$ peaks respectively of $\mathrm{NH}_{2}-\mathrm{SiO}_{2}-\mathrm{GO}$ microcapsules, (d \& e) $\mathrm{C}$ 1s and $\mathrm{Si} 2 \mathrm{p}$ peaks respectively of $\mathrm{SiO}_{2}-\mathrm{GO}$ microcapsules, ( $f \& g$ ) $C 1 s$ and $O 1 S$ peaks respectively of GO sheets. Symbols indicate experimental XPS data, and the solid lines are model fits for individual components. 


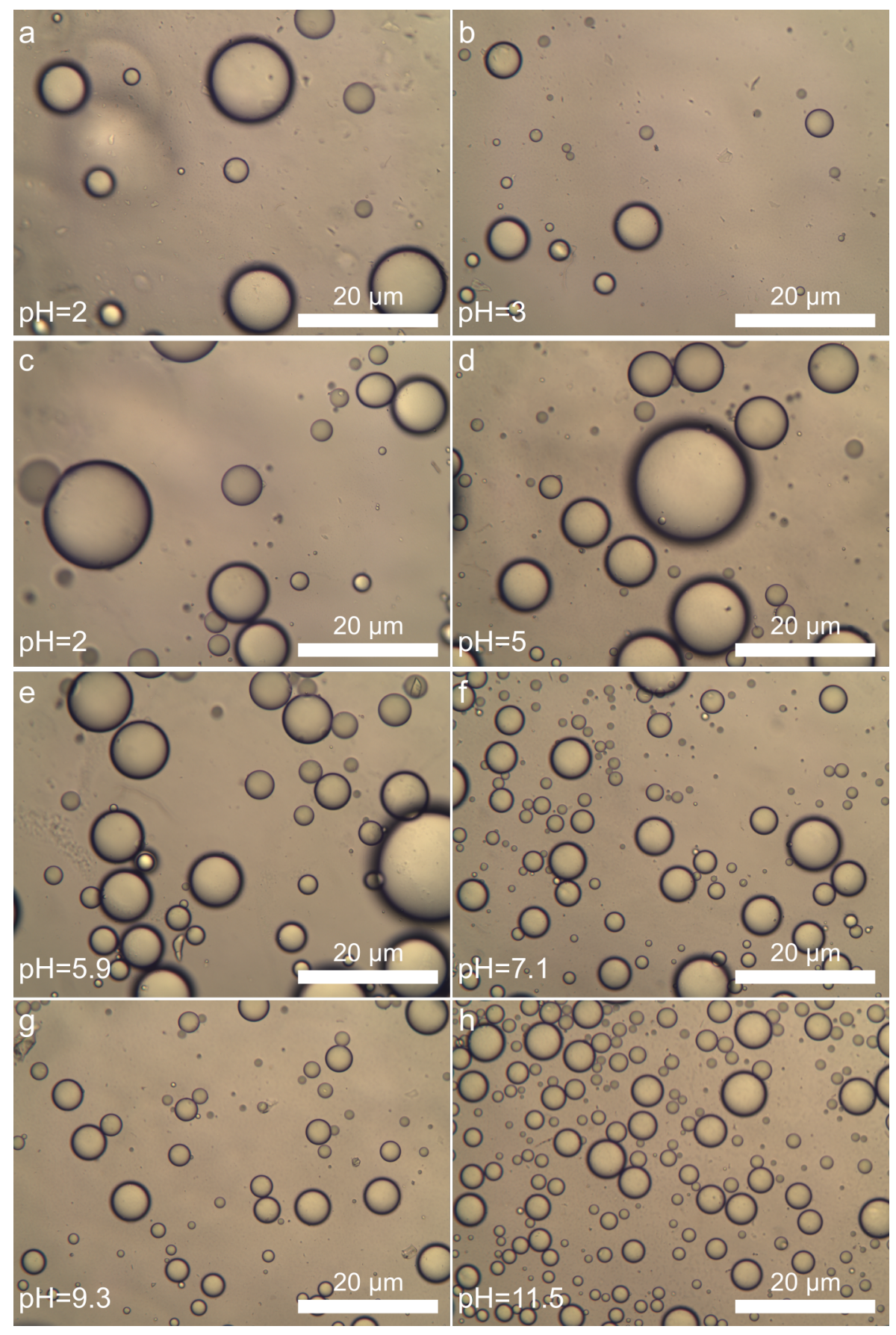

Figure S2. Photographs and optical microscopy images of $\mathrm{SiO}_{2}-\mathrm{GO}$ microcapsules prepared from GO-stabilized Pickering emulsions made using 20\% TEOS in toluene and $2 \mathrm{mg} / \mathrm{mL}$ GO in water at different $\mathrm{pHs}$, with a 1:1 volume ratio of oil:water. 


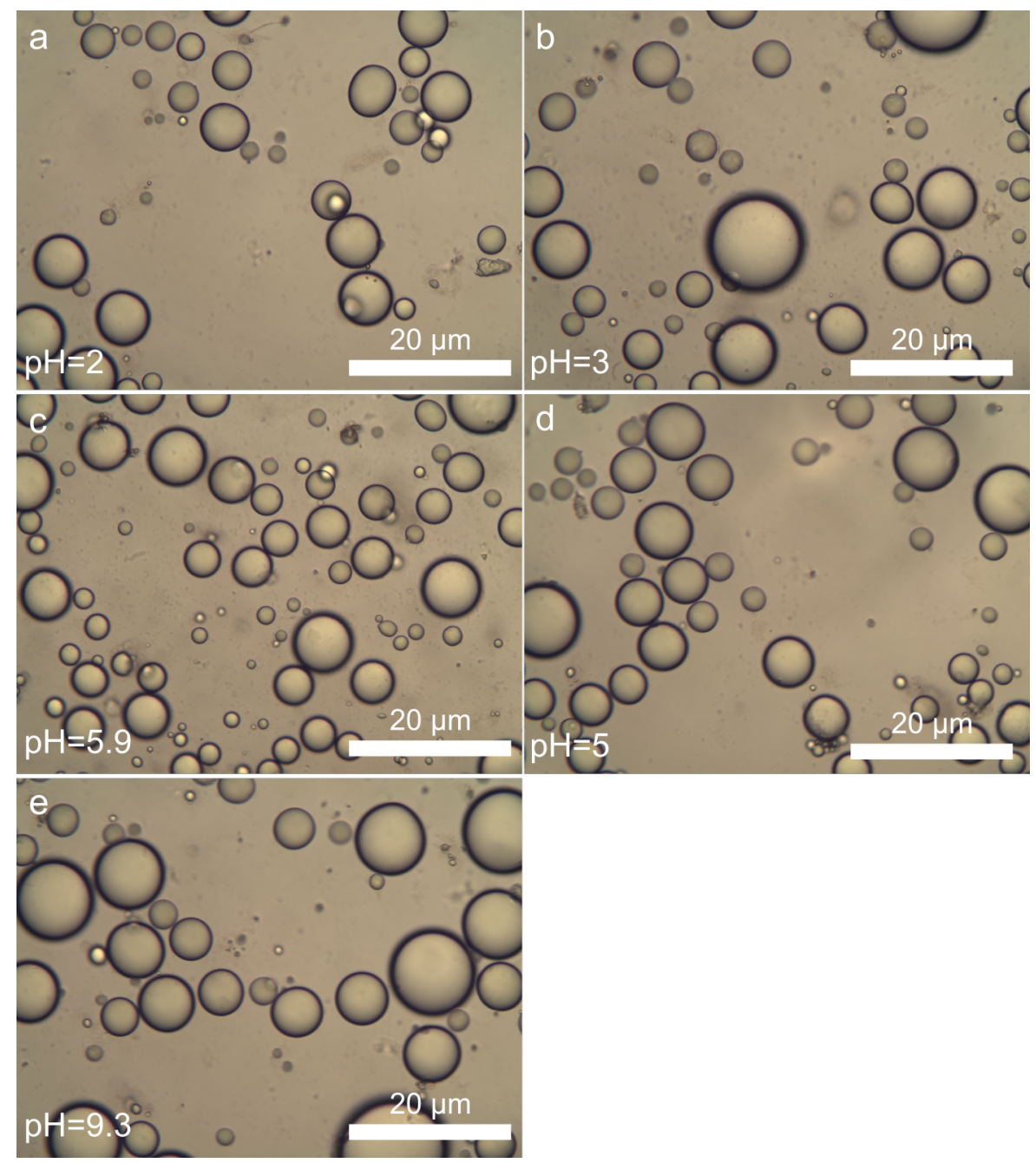

Figure S3. Photographs and optical microscopy images of $\mathrm{NH}_{2}-\mathrm{SiO}_{2}-\mathrm{GO}$ microcapsules obtained from GO-stabilized Pickering emulsions as Figure 2 above but with 0.027 wt. $\%$ added APTES.

Calibration curve of PFOA: A calibration curve was made using a wide range of PFOA concentrations from $20 \mathrm{mg} / \mathrm{L}$ to $600 \mathrm{mg} / \mathrm{L}$, and is shown in Fig S4, covering the desired concentration range under study, linking area under LC peak with the corresponding concentration. This could thereby be applied to determine unknown concentrations of PFOA. The trend shows a linear response with high $\mathrm{R}^{2}=0.998$. 


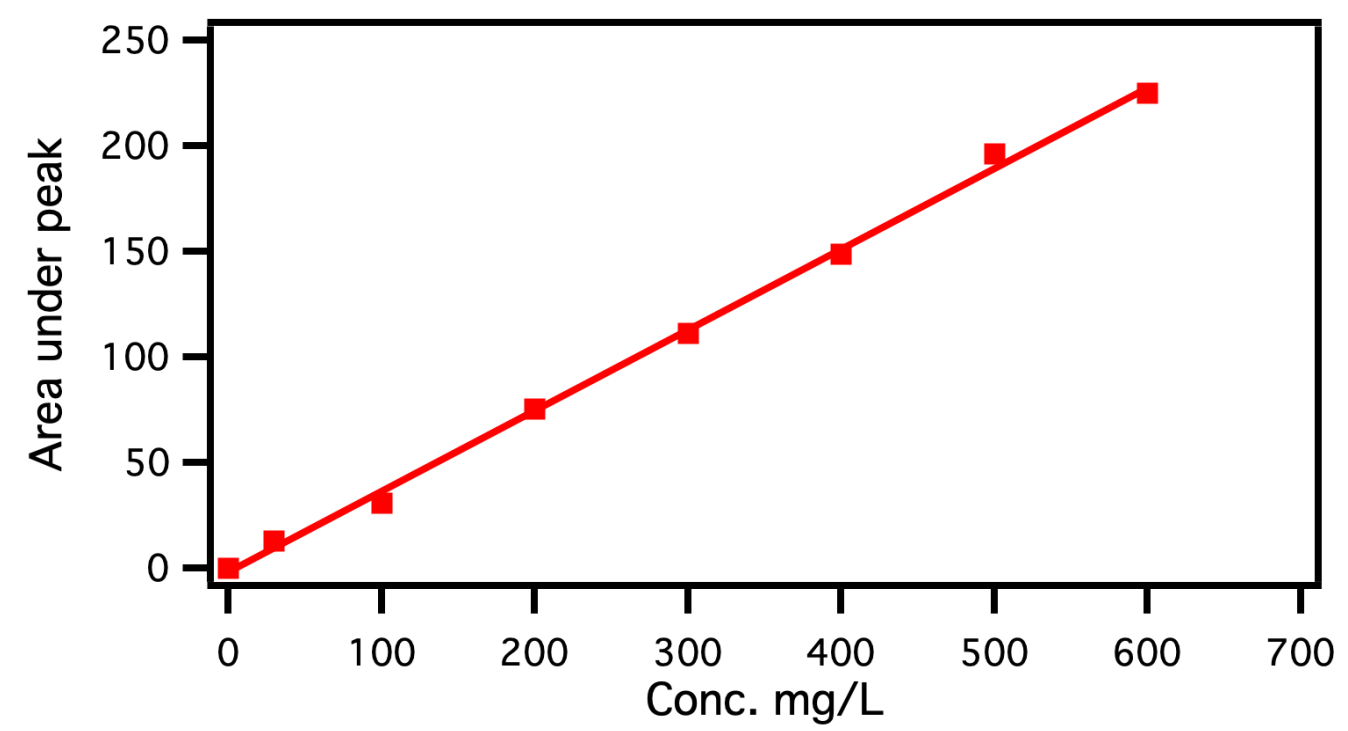

Fig. S4. Calibration curve for PFOA using a wide concentration range $(20 \mathrm{mg} / \mathrm{L}$ to $200 \mathrm{mg} / \mathrm{L}$ ); injection amount $5 \mu \mathrm{L}$ and detection wavelength $205 \mathrm{~nm}$.

Table S1. Tabulated data of corresponding area under peak for different concentrations of PFOA solution.

\begin{tabular}{|l|l|l|l|l|l|l|l|l|}
\hline $\begin{array}{l}\text { PFOA } \\
\text { concentration } \\
\text { mg/L }\end{array}$ & 0 & 30 & 100 & 200 & 300 & 400 & 500 & 600 \\
\hline $\begin{array}{l}\text { Area under } \\
\text { peak }\end{array}$ & 0.0 & 13.0 & 75.5 & 75.5 & 111.1 & 148.6 & 196.4 & 225.0 \\
\hline
\end{tabular}

Effect of initial PFOA concentration on adsorption efficiency of microcapsules:

To investigate the optimum efficiency of the PFOA adsorption procedure using microcapsules, adsorbed amount as a function of initial PFOA concentration ranging from $50 \mathrm{mg} / \mathrm{L}$ to $600 \mathrm{mg} / \mathrm{L}$ was monitored using HPLC (Fig. S2). 


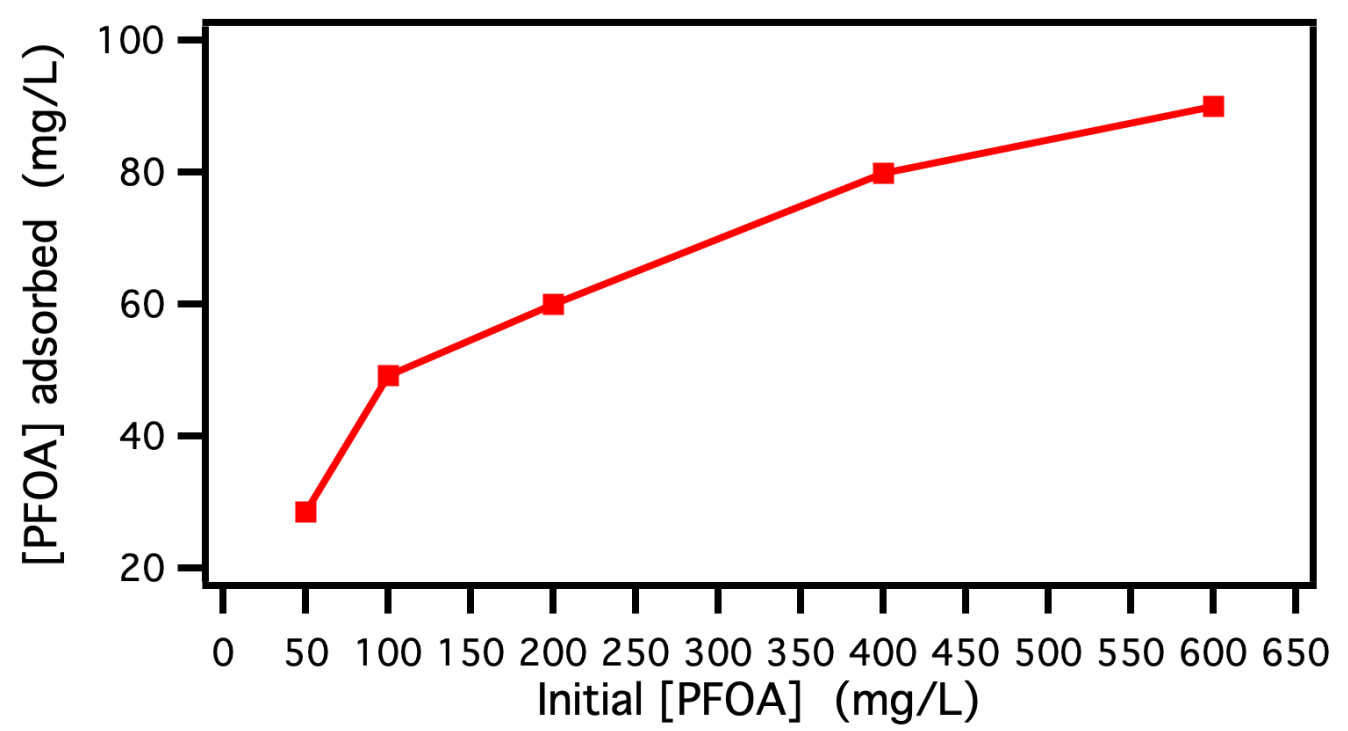

Figure S5. The adsorbed amount of PFOA by $\mathrm{NH}_{2}-\mathrm{SiO}_{2}-\mathrm{GO}$ microcapsules for different initial concentrations of PFOA.

Table S2. Tabulated data showing PFOA adsorbed for different initial concentrations of PFOA.

\begin{tabular}{|l|l|l|l|l|l|}
\hline $\begin{array}{l}\text { PFOA initial } \\
\text { concentration } \\
\mathrm{mg} / \mathrm{L}\end{array}$ & 50 & 100 & 200 & 400 & 600 \\
\hline $\begin{array}{l}\text { PFOA } \\
\text { adsorbed } \\
\mathrm{mg} / \mathrm{L}\end{array}$ & 28.6 & 49.2 & 60.0 & 79.9 & 90.0 \\
\hline
\end{tabular}

Effect of injection amount on detection procedure for PFOA: further experiments were conducted to investigate the sensitivity of the polymer column for PFOA. It was found that the adsorption of PFOA corresponding to the area under peak increased with decreasing injection amount, recording $33.8 \%$ and $31.0 \%$ at $5 \mu \mathrm{L}$ and $10 \mu \mathrm{L}$ injection amount respectively. This value decreased significantly to reach $12.7 \%$ at $50 \mu \mathrm{L}$ injection amount (Fig. S3 and table S3). In accordance with these data, the polymer column was used due to its high sensitivity for PFOA detection. 


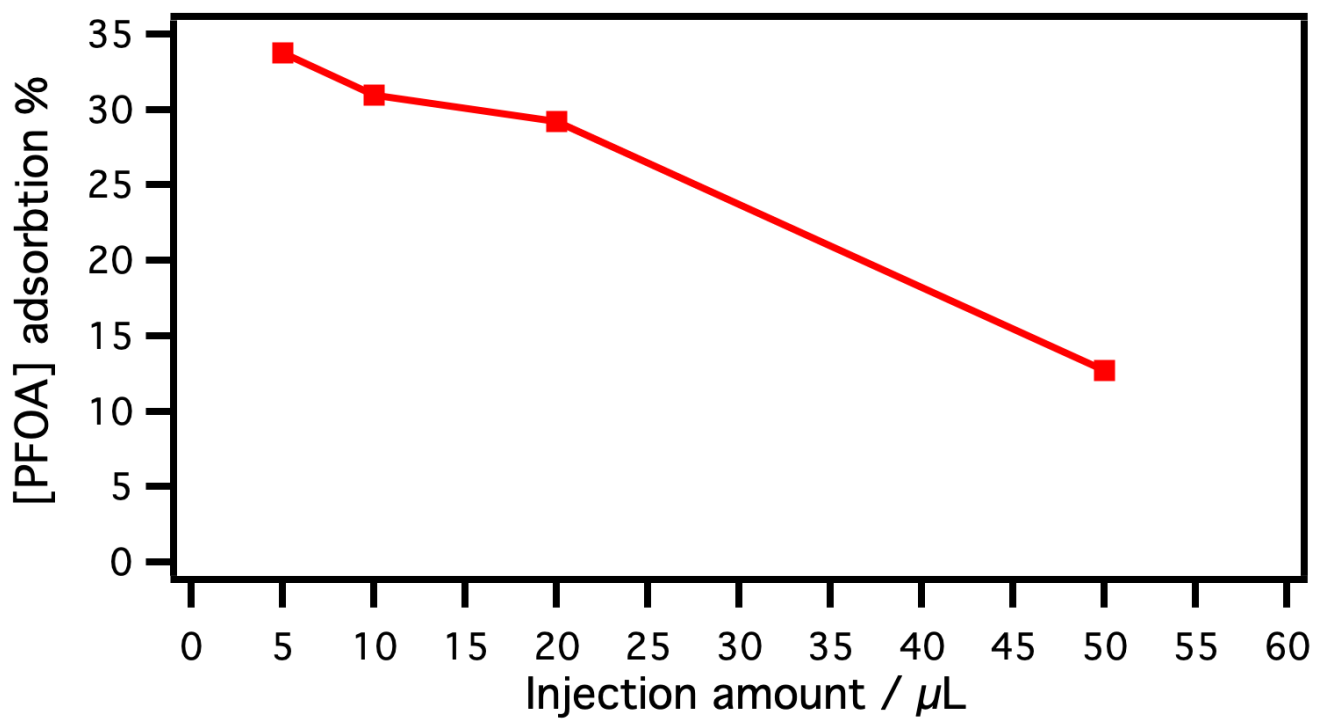

Figure S6. Effect of different injection amounts of $200 \mathrm{mg} / \mathrm{L}$ PFOA solution in water on area under peak value.

Table S3. Tabulated data indicating the relationship between injection amount of $200 \mathrm{mg} / \mathrm{L}$ PFOA solution of and adsorption \%.

\begin{tabular}{|l|l|l|l|l|}
\hline Injection amount / $\mu \mathrm{L}$ & 5 & 10 & 20 & 50 \\
\hline $\begin{array}{l}\text { Area under peak before } \\
\text { adsorption }\end{array}$ & 75.5 & 168.0 & 346.0 & 913.0 \\
\hline $\begin{array}{l}\text { Area under peak after } \\
\text { the adsorption }\end{array}$ & 50.0 & 116.0 & 245.0 & 797.0 \\
\hline Adsorption \% & 33.75 & 30.95 & 29.20 & 12.70 \\
\hline
\end{tabular}


Table S4. Partitioning quantification for $200 \mathrm{mg} / \mathrm{L}$ PFOA between olive oil and water at 1:1 mole ratio at different $\mathrm{pH}$ values as determined using HPLC, where AUP= Area under peak and $\mathrm{CC}=$ corresponding concentration.

\begin{tabular}{|l|l|l|l|l|l|l|l|l|l|l|}
\hline \multirow{2}{*}{$\begin{array}{c}\text { Area under } \\
\text { peak } \\
\text { Time/h }\end{array}$} & \multicolumn{3}{|c|}{$\mathrm{pH1}$} & \multicolumn{2}{c|}{$\mathrm{pH} 2$} & \multicolumn{2}{c|}{$\mathrm{pH}$} & \multicolumn{2}{c|}{$\mathrm{pH} 10$} \\
& AUP & CC & AUP & CC & AUP & CC & AUP & CC & AUP & CC \\
\hline 0 & 75.5 & 200.0 & 75.5 & 200.0 & 75.5 & 200.0 & 75.5 & 200.0 & 75.5 & 200.0 \\
\hline 2 & 14.0 & 37.1 & 42.0 & 111.3 & 57.0 & 151.0 & 60.4 & 160.0 & 24.0 & 63.6 \\
\hline 5 & 0.0 & 0.0 & 0.0 & 0.0 & 54.0 & 143.0 & 54.7 & 145.0 & 32.0 & 84.8 \\
\hline 9 & 0.0 & 0.0 & 0.0 & 0.0 & 42.0 & 111.3 & 45.3 & 120.0 & 59.0 & 156.3 \\
\hline 17 & 0.0 & 0.0 & 0.0 & 0.0 & 31.0 & 82.1 & 34.0 & 90.0 & 93.0 & 246.4 \\
\hline 24 & 0.0 & 0.0 & 0.0 & 0.0 & 23.0 & 60.9 & 28.3 & 75.0 & 98.0 & 259.6 \\
\hline
\end{tabular}

Table S5. Effect of $\mathrm{pH}$ on PFOA adsorption by $\mathrm{NH}_{2}-\mathrm{SiO}_{2}-\mathrm{GO}$ capsules at 1:1 olive oil: water mole ratio, where $\mathrm{AUP}=$ Area under peak and $\mathrm{CC}=$ corresponding concentration.

\begin{tabular}{|c|c|c|c|c|c|c|}
\hline \multirow{2}{*}{$\begin{array}{l}\text { Area under } \\
\text { peak }\end{array}$} & \multicolumn{3}{|c|}{$\mathrm{pH} 2$} & \multicolumn{3}{|c|}{$\mathrm{pH} 5$} \\
\hline & AUP & $\begin{array}{l}\mathrm{CC}(\mathrm{mg} / \mathrm{L}) \text { of } \\
\text { remaining } \\
\text { PFOA }\end{array}$ & $\begin{array}{l}\mathrm{CC}(\mathrm{mg} / \mathrm{L}) \text { of } \\
\text { Adsorbed } \\
\text { PFOA }\end{array}$ & AUP & $\begin{array}{l}\mathrm{CC}(\mathrm{mg} / \mathrm{L}) \text { of } \\
\text { remaining } \\
\text { PFOA }\end{array}$ & $\begin{array}{l}\mathrm{CC}(\mathrm{mg} / \mathrm{L}) \text { of } \\
\text { Adsorbed } \\
\text { PFOA }\end{array}$ \\
\hline 0 & 75.5 & 200.0 & 0.0 & 75.5 & 200.0 & 0.0 \\
\hline $30 \mathrm{~m}$ & 44.0 & 116.6 & 83.4 & 56.0 & 148.3 & 51.7 \\
\hline $1.0 \mathrm{~h}$ & 40.0 & 106.0 & 94.0 & 50.0 & 132.5 & 67.5 \\
\hline $1: 30 \mathrm{~h}$ & 37.0 & 98.01 & 102.0 & 50.0 & 132.5 & 67.5 \\
\hline $2 \mathrm{~h}$ & 35.0 & 92.7 & 107.3 & 46.0 & 121.9 & 78.1 \\
\hline $4 h$ & 32.8 & 84.8 & 115.2 & 44.0 & 116.6 & 83.4 \\
\hline $6 h$ & 29.0 & 76.8 & 123.2 & 39.0 & 103.3 & 96.7 \\
\hline $8 \mathrm{~h}$ & 25.0 & 66.2 & 133.8 & 36 & 95.4 & 104.6 \\
\hline $17 \mathrm{~h}$ & 9.0 & 23.8 & 176.2 & 23.0 & 60.9 & 139.1 \\
\hline $24 \mathrm{~h}$ & 7.0 & 18.5 & 181.5 & 20.0 & 53.0 & 147.0 \\
\hline 2 days & 0.0 & 0.0 & 200.0 & 13.0 & 34.4 & 165.6 \\
\hline 3 days & 0.0 & 0.0 & 200.0 & 11.0 & 29.1 & 170.9 \\
\hline $\begin{array}{l}\text { Breaking } \\
\text { capsule }\end{array}$ & 73.8 & 195.5 & 4.5 & 78.5 & 208.0 & 0.0 \\
\hline
\end{tabular}


Table S6. Effect of $\mathrm{pH}$ on the PFOA adsorption by $\mathrm{SiO}_{2}-\mathrm{GO}$ capsules at 1:1 olive oil: water mole ratio, where $\mathrm{AUP}=$ Area under peak and $\mathrm{CC}=$ corresponding concentration.

\begin{tabular}{|c|c|c|c|c|c|c|}
\hline Area under & & $\mathrm{pH} 5$ & & & $\mathrm{pH} 2$ & \\
\hline Time & AUP & $\begin{array}{l}\mathrm{CC}(\mathrm{mg} / \mathrm{L}) \text { of } \\
\text { remaining } \\
\text { PFOA }\end{array}$ & $\begin{array}{l}\mathrm{CC}(\mathrm{mg} / \mathrm{L}) \text { of } \\
\text { Adsorbed } \\
\text { PFOA }\end{array}$ & AUP & $\begin{array}{l}\mathrm{CC}(\mathrm{mg} / \mathrm{L}) \text { of } \\
\text { remaining PFOA }\end{array}$ & $\begin{array}{l}\text { CC }(\mathrm{mg} / \mathrm{L}) \text { of } \\
\text { Adsorbed } \\
\text { PFOA }\end{array}$ \\
\hline 0 & 75.5 & 200 & 0 & 75.5 & 200 & 0 \\
\hline $1 \mathrm{~h}$ & 67.1 & 177.7 & 22.3 & 69.9 & 185.1 & 14.9 \\
\hline $2 \mathrm{~h}$ & 72.3 & 191.5 & 8.5 & 70.3 & 186.2 & 13.8 \\
\hline $3 \mathrm{~h} 30 \mathrm{~min}$ & 75.3 & 199.5 & 0.5 & 69.9 & 185.1 & 14.9 \\
\hline $4 \mathrm{~h} 15 \mathrm{~min}$ & 72.8 & 192.8 & 7.2 & 67.4 & 178.5 & 21.5 \\
\hline $6 \mathrm{~h}$ & 73.1 & 193.6 & 6.4 & 61 & 161.6 & 38.4 \\
\hline $8 \mathrm{~h}$ & 73.5 & 194.7 & 5.3 & 62.1 & 164.5 & 35.5 \\
\hline $21 \mathrm{~h}$ & 66.1 & 175.0 & 25 & 61.8 & 163.7 & 36.3 \\
\hline $24 \mathrm{~h}$ & 62.9 & 166.6 & 33.4 & 60.6 & 160.5 & 39.5 \\
\hline 2 days & 73.6 & 194.9 & 5.1 & 47 & 124.5 & 75.5 \\
\hline 3 days & 72 & 190.7 & 9.3 & 45.3 & 120 & 80 \\
\hline $\begin{array}{l}\text { Breaking } \\
\text { Capsule }\end{array}$ & 77.4 & 205 & 0 & 74.6 & 197.5 & 2.5 \\
\hline
\end{tabular}




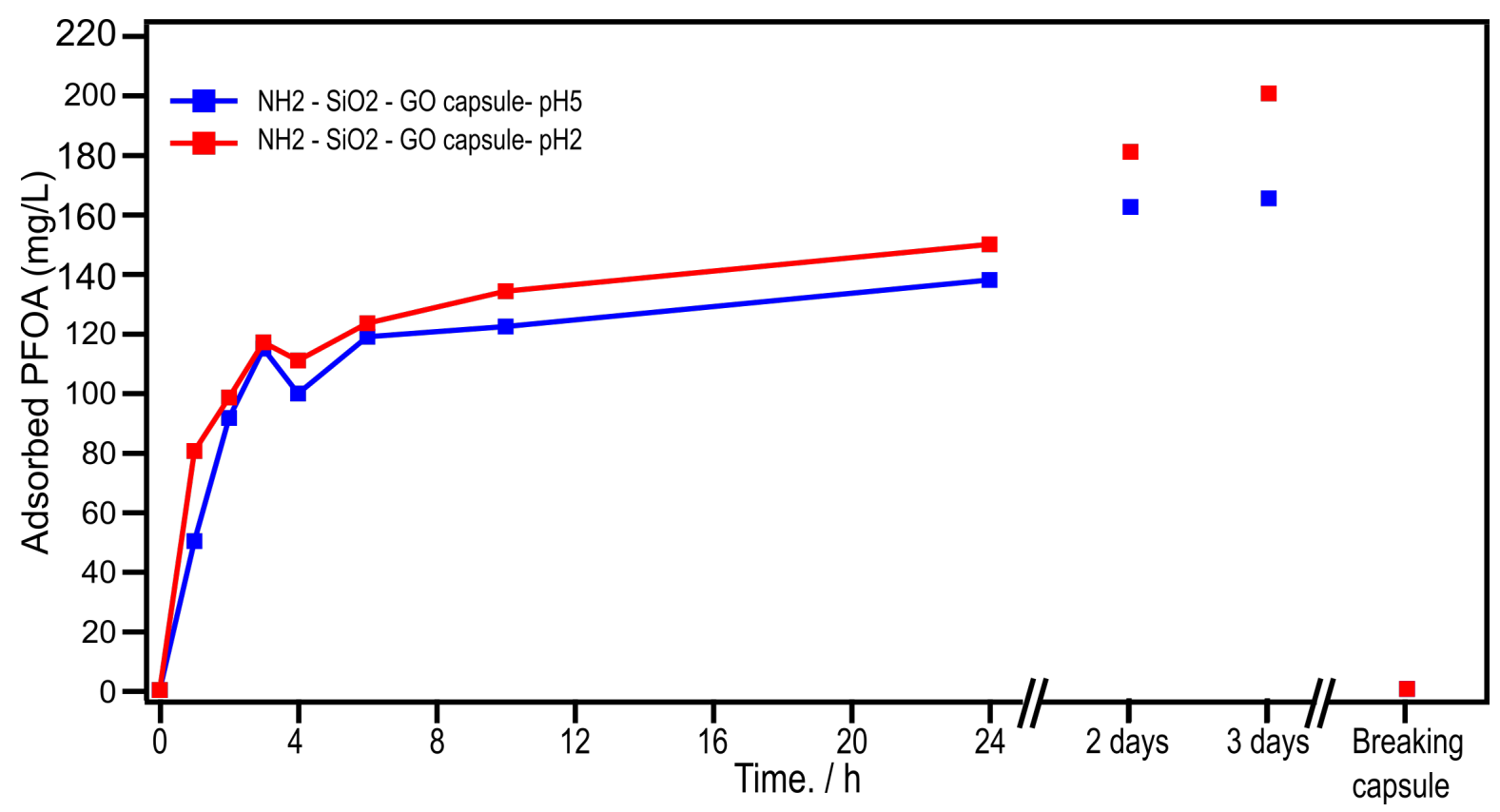

Figure S7. Effects of pH on PFOA adsorption by $\mathrm{NH}_{2}-\mathrm{SiO}_{2}-\mathrm{GO}$ microcapsules prepared using 2:1 volume ratio of olive oil: water.

Table S7. Effect of $\mathrm{pH}$ on PFOA adsorption by $\mathrm{NH}_{2}-\mathrm{SiO}_{2}-\mathrm{GO}$ capsules synthesised using a 2:1 olive oil: water volume ratio, where $\mathrm{AUP}=$ Area under peak and $\mathrm{CC}=$ corresponding concentration.

\begin{tabular}{|l|l|l|l|l|l|l|}
\hline $\begin{array}{r}\text { Area under } \\
\text { peak }\end{array}$ & \multicolumn{3}{|c|}{ pH 5 } & \multicolumn{3}{c|}{ pH 2 } \\
\cline { 2 - 7 } & AUP & $\begin{array}{l}\text { CC (mg/L) } \\
\text { of } \\
\text { remaining } \\
\text { PFOA }\end{array}$ & $\begin{array}{l}\text { CC(mg/L) of } \\
\text { Adsorbed } \\
\text { PFOA }\end{array}$ & AUP & $\begin{array}{l}\text { CC (mg/L) } \\
\text { of } \\
\text { remaining } \\
\text { PFOA }\end{array}$ & $\begin{array}{l}\text { CC (mg/L) } \\
\text { of Adsorbed } \\
\text { PFOA }\end{array}$ \\
\hline 0 & 75.5 & 200 & 0 & 75.5 & 200 & 0 \\
\hline $1 \mathrm{~h}$ & 56.6 & 150.0 & 50 & 45.2 & 119.7 & 80.3 \\
\hline $2 \mathrm{~h}$ & 41.0 & 108.6 & 91.4 & 38.4 & 101.7 & 98.3 \\
\hline $3 \mathrm{~h}$ & 32.2 & 85.3 & 114.7 & 31.4 & 83.2 & 116.8 \\
\hline $4 \mathrm{~h}$ & 37.9 & 100.4 & 99.6 & 33.7 & 89.3 & 110.7 \\
\hline $6 \mathrm{~h}$ & 30.7 & 81.3 & 118.7 & 29.0 & 76.8 & 123.2 \\
\hline $10 \mathrm{~h}$ & 29.4 & 77.9 & 122.1 & 24.9 & 66.0 & 134 \\
\hline $24 \mathrm{~h}$ & 23.5 & 62.3 & 137.7 & 19.0 & 50.3 & 149.7 \\
\hline 2 2days & 14.4 & 38.1 & 161.9 & 7.4 & 19.6 & 180.4 \\
\hline 4 days & 13.3 & 35.2 & 164.8 & 0 & 0 & 200 \\
\hline $\begin{array}{l}\text { Breaking } \\
\text { Capsule }\end{array}$ & & 201 & 0 & & 206 & 0 \\
\hline
\end{tabular}


LC-MS analysis for PFOA adsorption: LC-MS was exploited to measure the decrease of PFOA concentration, after adsorption by microcapsules. Fig. S8 shows the MS signals of PFOA residuals in solution, and the results demonstrate the strong and sharp main signal (red line) $412(\mathrm{~m} / \mathrm{z})$ corresponding to PFOA, with some weak signals at around 350 and $370(\mathrm{~m} / \mathrm{z})$, likely to indicate either PFOA isomerism or contamination.

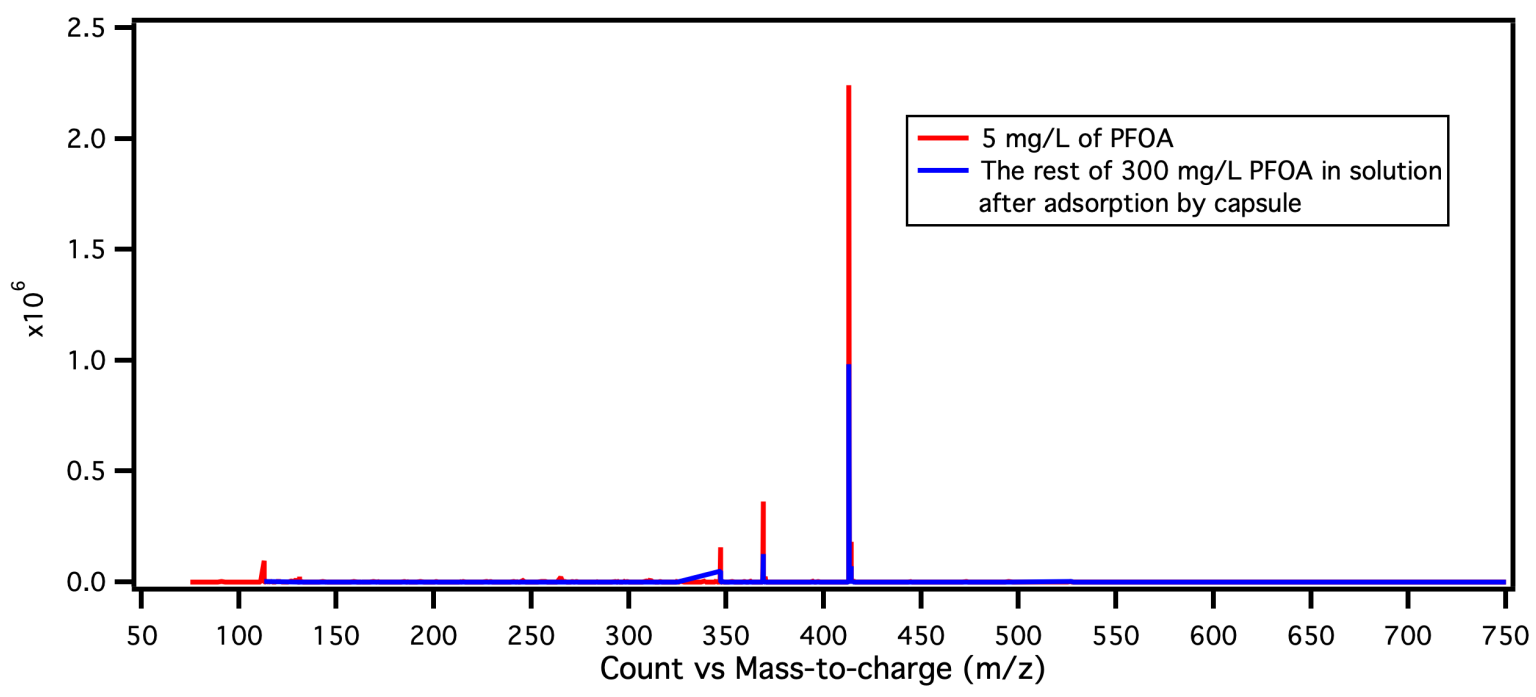

Fig. S8. Mass spectra (MS) of $5 \mathrm{mg} / \mathrm{L}$ PFOA solution (red trace) and the remaining PFOA after adsorption by $\mathrm{NH}_{2}-\mathrm{SiO}_{2}-\mathrm{GO}$ microcapsules from an initial PFOA concentration of $300 \mathrm{mg} / \mathrm{L}$ (blue trace). 